\title{
PENSAMIENTO COMPLEJO DE EDGAR MORIN: NUEVA VISIÓN DEL CONOCIMIENTO
}

\section{EDGAR MORIN'S COMPLEX THOUGHT: NEW LOOK ON THE KNOWLEDGE}

\author{
${ }^{a}$ Esp. Juan Pablo Peñaranda Franco \\ ${ }^{a}$ Universidad Francisco de Puala Santander Ocaña, Grupo de Investigación de la Facultad de \\ Educación, Artes y Humanidades GIFEAH, Vía Acolsure, Sede el Algodonal, Ocaña - Norte de \\ Santander, Colombia, jppenarandaf@ufpso.edu.co
}

Fecha de recepción: 24-10-2016

Fecha de aprobación: 11-30-2016

\begin{abstract}
Resumen: el pensamiento complejo de Edgar Morin es un referente que se instaura como un intento de mediación para la comprensión de los procesos intelectivos que exige la sociedad de conocimiento del siglo XXI. En el seno del mismo la integración del mayor número de facetas es importante si el sujeto del conocimiento desea abordar a un objeto específico. Sabiendo que en el fondo el proceso de conocimiento es mediado por todas las dimensiones constitutivas del hombre, se debe comprender que el mismo intento de conocer lleva en el seno incertidumbres, aleatoreidades, azares, errores junto a las certezas, determinismos y simplicidades propias incluso de la dimensión biológica de la que surge el pensamiento.
\end{abstract}

Palabras clave: complejidad, computación, conocimiento, ruido informacional.

\begin{abstract}
Edgar Morin's complex thought is a benckmarch that is established as an attempt to mediate the understanding of intellective processes that the knowledge society of XXI century, requires. Within, the integration as many facets, it's important if the knowledge subject wishes to address a specific object. Knowing that knowledge process is mediated by all the men's constitutive dimensions, must understand that the attempt to meet carries within uncertainties, aleatoriety, hazards, errors with certainty, determinism and own simplicities even biological dimension from the thought arises
\end{abstract}

Keywords: complexity, computation, informational noise, knowledge. 


\section{INTRODUCCIÓN}

El camino de las ciencias ha transitado el sendero que la epistemología ha trazado durante siglos. La visión que este ejercicio de reflexión filosófica ha brindado a la ciencia luces y sombras; desafíos y potencialidades.

Edgar Morin, pensador de este tiempo hace una denuncia de las limitaciones que el ejercicio epistemológico ha brindado a las ciencias y que constituye en esencia sus propias limitaciones: la construcción de un concepto de verdad que se constituye en garantía de la edificación de certezas. La complejidad brinda un marco para repensar estas edificaciones del conocimiento; si en el seno de cualquier proceso habitan los errores, las aleatoreidades, la incertidumbre ¿cómo pensar en un producto que no esté exento de estos constitutivos? El presente artículo se pregunta por estos aspectos.

\section{GENERALIDADES DEL PENSAMIENTO COMPLEJO DE EDGAR MORIN}

Edgar Morin, pensador francés, actualmente ha abierto las puertas a una nueva manera de ver el mundo, de asumir la realidad: el pensamiento complejo. Surge de manera inicial una primera pregunta que ayuda a comprender el hecho puntual que se está desarrollando ¿qué es la complejidad? Complejo es todo aquello que está constituido por una diversidad de aspectos a analizar con dificultad de comprender o resolver.

Desde un punto de vista etimológico la palabra "complejidad" es de origen latino, proviene de "complectere", cuya raíz "plectere" significa trenzar, enlazar (...) El agregado "com" añade el sentido de la dualidad de dos elementos opuestos que enlazan íntimamente, pero sin anular su dualidad. (Morin, Ciurana, \& Motta, 2002 p. 40)

Siguiendo el rastro dejado, se hace necesario abordar la complejidad como todo aquello que es intrincado, que aparece oculto a la simple mirada holística que lo categoriza todo; es todo aquello que aparece y genera incertidumbre porque va más allá, no se queda ni en el todo ni en las partes -embucla estas dos realidades-; complejidad por tanto se puede entender como un camino hacia lo complejo.

Morin establece un estatuto que ayuda a vislumbrar el horizonte característico del pensamiento complejo:

1. Hay múltiples vías de entrada a la complejidad; diferentes autores utilizan el término de forma diversa.

2. Los autores citados anteriormente distinguen entre complejidad y complicación, puntos de vista simplemente cuantitativos y cualitativos. El discurso sobre la complejidad es lógico y general; concierne no solo a la ciencia sino a la sociedad, la ética y la política. Concierne a una epistemología general. El mismo se crea y recrea en el mismo caminar.

3. El pensamiento complejo sabe que la certidumbre generalizada es un mito y es más potente un pensamiento que reconoce la vaguedad y la imprecisión que un pensamiento que la excluye irreflexivamente.

4. El pensamiento complejo nunca es un pensamiento completo; es un pensamiento articulante y multidimensional. $\mathrm{Su}$ ambición es articular fragmentos disciplinarios antagónicos fracturados por el pensamiento simplificador. Hay una 
imposibilidad, incluso teórica de la omnisciencia. Así como también reconoce el estado transitorio y cuasi esquemático de todo concepto. Implica un principio de incompletud e incertidumbre.

5. El pensamiento complejo reconoce dos tipos de ignorancia: la del que no sabe y quiere aprender y la de quien cree que el conocimiento es un proceso lineal, acumulativo, que avanza haciendo luz allí donde antes había oscuridad, ignorando que toda luz produce, como efecto, sombras.

6. El pensamiento complejo no desprecia lo simple, critica la simplificación. No se trata de poner de cabeza a la simplificación sino la unión de la misma con la complejidad. El pensamiento complejo no excluye, integra (1999 p. 47-53).

Surge una pregunta que puede ser natural realizarse luego de las anteriores consideraciones ¿qué es la simplicidad que el pensamiento complejo critica? El mismo Morin realiza una reflexión al expresar que la simplicidad es un paradigma que tiende a reconocer en el orden el principio generador de conocimiento. Traslapando estos conceptos al mundo científico, se puede corroborar sin lugar a dudas que el método científico ha sido el juez inquisitorial para la consideración de lo que es y lo que no puede llegar a ser conocimiento. Se desestima de esta forma al error, al desorden, a lo aleatorio, lo azaroso. Pero ¿cómo es posible que esto suceda? ¿Por qué es conocimiento la construcción humana que está libre de error y aleatoreidad si en la base, el hombre utiliza mecanismos falibles para llegar a estos constructos? (2001)

Allí donde la simplicidad ha olvidado el papel del desorden, donde ha legado dilemas disyuntores y reductores (en la vida cotidiana) es pertinente disipar los fantasmas, las sombras que adormecen el criterio del hombre pues la disyunción de las ciencias ha perdido al hombre como sujeto individual y lo ha insertado en un mundo de pluralidad. En este sentido, la vida humana, siendo las dos cosas al tiempo (experiencia individual y fenómeno social) acontece con una multiplicidad abrumadora de dimensiones desde la cual puede entenderse: aparece la dimensión biológica, anatómica, fisiológica, cultural, psicológica; inmersa en un universo ideológico, lingüístico, conciencial... todas ellas, al mismo tiempo. Se requiere por tanto abogar por un paradigma que no reduzca, que no simplifique sino que ayude a ligar las realidades que los modelos tradicionales han separado y que separen las que ha unido.

López Ramírez amplía el horizonte considerando por esta vía de integración, de conocimiento, de desconocimiento que es la complejidad, mencionando que parece que los individuos, en medio de la incertidumbre del conocimiento humano, van perdiéndose; sin embargo, éste es un buen camino, es una buena señal, pues de esta derivación de incertidumbres, es en donde el conocimiento se va construyendo. Si el conocimiento se cimentara sobre certezas absolutas, no habría necesidad de seguir indagando; sin embargo, la realidad abordada desde las ciencias, aparece como incompleta, pues ninguna ciencia, ningún método puede llegar a apropiarse de verdades absolutas. Este es el problema que en el fondo el dogmatismo revela: la confianza plena en la posibilidad de conocer; en suma, no es más que la aceptación de una posesión absoluta del objeto por parte del sujeto. La práctica misma ayuda a despejar que solamente el sujeto puede llegar a conocer aspectos aislados del objeto, a pesar de la fuerte vocación del hombre por llegar a la verdad. La complejidad reconoce que este llamado 
es un reconocimiento de las incertidumbres implícitas en el proceso de conocimiento, por tanto, el hombre, al saberse en la ignorancia, requiere abordar el objeto desde diversos ángulos, debe abrazar la transdisciplinariedad y por tanto su itinerario es inacabado (1998)

\section{LA COMPLEJIDAD EN EL CONOCIMIENTO}

\subsection{La máquina hipercompleja}

No se puede considerar el proceso de conocimiento, sin realizar una mirada holística al menos del órgano del conocimiento que es el cerebro: la gran máquina que ha hecho la diferencia evolutiva, la gran estructura por la cual el hombre es lo que es. Pero ¿por qué se dice que el cerebro no solamente es una máquina sino que además es hipercompleja? Para vislumbrar la respuesta del anterior interrogante se podría hacer un simple ejercicio pensando en el grandioso, incontable número de interacciones que las partes y el todo (de este enigmático órgano) establecen entre sí. (Morin, 1999).

La complejidad cerebral va incluso más allá, pues el mismo, actúa en función de un programa que recoge en el seno mismo de su constitución, la facultad de tratar con el orden y el desorden, de tratar con la certidumbre y la incertidumbre, de tratar con la verdad y el error. Y más allá de estos umbrales de abstracción, como estructura tiene un grado de plasticidad tal, que en caso de ciertas lesiones, se reordena constitutivamente para seguir el funcionamiento de todo el sistema vivo.

Como unidad funcional del cerebro contamos con la neurona, célula capacitada para la transmisión de microimpulsos eléctricos a través de su membrana e incluso hacia neuronas contiguas. Como tal la neurona, por sí misma no es capaz de realizar un análisis estadístico, por ejemplo, pero en conjunto, el cerebro sí (si el individuo se ha formado para ello). Para lograrlo se requieren grandiosos niveles de relación interneuronal, de funciones histológicas, orgánicas.

Córdoba García ayuda a entender el hecho de que así como son complejas el número de relaciones abstractas que el cerebro puede establecer en relación a los conceptos, ideas... tisularmente es un tejido en el cual aisladamente cada célula, puede establecer relaciones sinápticas con otras neuronas en el orden del millón, tanto en su cercanía, como en zonas aisladas del sistema nervioso. Cada relación sináptica puede también establecerse, por medio de neurotransmisores específicos según la ubicación celular y su función (sea motora, sensitiva o visceral) para la cual está destinada, gatillada por la influencia de hormonas específicas. Especialista de la transmisión de impulsos (tanto químicos, gaseosos, como de potenciales de acción transmembrana), la neurona es la base funcional del cerebro y es la microestructura que ha permitido el desarrollo de la ciencia y la técnica por sus propiedades integrativas (2005).

Considerar el conocimiento sin despejar la fórmula del aspecto biológico que se encuentra presente en éste, es una limitante de nuestra concepción del mismo, pues vale la pena divisar las relaciones funcionales que pueden afincarse en este órgano tan privilegiado, para llegar a la conclusión de las limitaciones que el hombre tiene para llegar a consolidar paradigmas que se constituyan en certeza absoluta, debido a los umbrales constitucionales que median la aparición de ruidos informacionales a la hora de abstraer y categorizar cualquier fenómeno en unidades mentales abstractas. Se puede ofrecer como ejemplo la aplicación 
de un fuerte estímulo olfativo como en el caso de amoniaco o ciertos ácidos carboxílicos en las células de los nervios olfativos; puede verse luego de la estimulación un gran pico de actividad que luego de algunos instantes pueden llegar a valores mínimos e incluso equivalentes a cero, hasta que la membrana neuronal de estos centros sensitivos vuelve a repolarizarse.

Otro sencillo ejemplo sería pensar en la posibilidad de existencia de un ser bidimensional y se le preguntase acerca de la posibilidad de existencia de una tercera dimensión; seguramente éste aseguraría que esta petición es inconcebible, pues solo cabe en sus esquemas explicativos la existencia de dos dimensiones. Del mismo modo ha sucedido con el hombre a través de las épocas: ha temido pensar en lo impensable, de desafiar las fronteras de la realidad o simplemente ha endiosado sus propias concepciones. Verbo y gracia los fenómenos ocurridos con las ideologías del Partido Nacional Socialista de Alemania, que basadas en los principios de la selección natural, solo trajeron en el seno de las sociedades europeas destrucción y muerte durante la segunda guerra mundial.

\subsection{Resignificación del concepto de verdad}

Son muchas las voces que definen la verdad como la adecuación de la realidad con el intelecto (adequatio res intellectum) (Tarski, s.f.) ¿Puede el intelecto adecuarse a todas las facetas que nos ofrece la realidad? Se puede notar como primera medida un aspecto: adecuación vista desde los conceptos de orden y desorden, según los planteamientos deterministas, cae sin lugar a dudas en una posición privilegiada de orden; de ordenamiento sistemático de las esencias abstraidas del objeto real, que en el sujeto dejan una impresión intelectiva de éste y que lo llevan solamente a definirlo en virtud de él mismo.

Este tratamiento informacional se realiza en el cerebro "Los procesos fundamentales de la vida están en la vida, el pensamiento no puede ir más allá" (Husserl en Dúc Tháo, 1959 p. 140) De esta manera, el conocimiento está sujeto a la fisiología cerebral, que se definió de manera holística, como proceso complejo que integra realidades diversas dentro de un proceso computacional. Morin ayuda a entender la computación cerebral diciendo que la misma es un "complejo organizador/productor de carácter cognitivo que comporta una instancia informacional, una instancia simbólica, una instancia memorial y una instancia logicial” (1999, p. 47-48).

Conocer se traduce en computar, más que en adecuar la intelección a lo real pues este proceso es incompleto

Dicho de otro modo, el conocimiento necesariamente es: traducción en signos/símbolos, y sistemas de signos/símbolos (después con los desarrollos cerebrales, en representaciones, ideas, teorías...); construcción, es decir traducción constructora a partir de principios/reglas (logiciales) que permiten construir sistemas cognitivos que articulan información/signos/símbolos; solución de problemas, empezando por el problema cognitivo de la adecuación de la construcción traductora a la realidad que se trata de conocer. Es decir que el conocimiento no podría reflejar directamente lo real, no puede sino traducirlo y reconstruirlo en otra realidad (Morin, 1999, p. 59). 
De este modo la verdad debe ser redefinida y está subordinada a lo que de este proceso se pueda obtener.

\subsection{Ruido sensorio-informacional-lógico- memorial-simbólico: computación}

Los sistemas vivos, en las relaciones que deben establecer con el entorno en el cual habitan, se comprenden como sistemas autoorganizados (por organización se entiende a la posibilidad sistémica de un organismo de establecer inter-relaciones que hacen de éste un todo con posibilidad generadora de sí mismo). Wheatley \& Kellner Rogers, expresan sobre este aspecto:

Parte de la viabilidad proviene de la capacidad interna para crear estructuras para adecuarse al momento. Ni la forma ni la función (estructura y procesos) por sí mismo dicta cómo es construido el sistema... El sistema posee la capacidad para generar estructuras emergentes, dependiendo de lo que es requerido. NO está cerrada en una sola forma sino que es capaz de organizar información en la estructura que mejor cumpla con la necesidad presente. (...) Aunque flexible, una estructura auto-organizaa no es un reactor pasivo a fluctuaciones. Cuando madura y se estabiliza, llega a ser más eficiente en el uso de sus recursos y mejor capacitado para existir dentro de su medio ambiente. Establece una estructura básica que da soporte al desarrollo del sistema. Esta estructura entonces facilita la formación de un aislamiento del medio ambiente que protege al sistema de cambios constantes y reactivos (1998, pag. 90 y 92).

La consideración sistémica involucra el aspecto informacional como eminentemente constitutivo de su estructura (del evento vivo). Y donde hay información, el ruido (entendido como cualquier perturbación a la hora de emitirse la información, como en el mismo proceso de transmisión, recepción y tratamiento de ésta) está presente. Si se entiende el proceso de pensamiento como una computación viva ¿cuántas perturbaciones no tedrá este proceso al integrar tantos elementos al mismo tiempo? A un órgano hipercomplejo, no le quedan más sino acciones hipercomplejas y es allí, en el seno de estas complejidades, en donde se desenvuelve la trama por la lucha de la verdad por serlo y del error por descartarse del proceso de conocimiento visto desde el determinismo propio del paradigma de la simplicidad.

El ruido da como resultado, producto de las perturbaciones, más complejidad; en otras palabras, incertidumbres, indeterminaciones; ya que una información de la realidad trastocada ella misma (como en el caso de una percepción que se limita como se mencionó a la fisiología neuronal individualmente y nerviosa en su conjunto y como tal se limita a los umbrales de estas estructuras) encuentra en el fondo del proceso cognitivo principios lógicos inciertos, datos almacenados insuficientes o alterados y representaciones simbólicas pobres.

Estos elementos tendrán entonces una traducción/construcción/solución

problemática en la misma medida, no muy fiel al objeto, una intelección no adecuada a la realidad sino más bien próxima a ésta; en una medida u otra según lo dicho, habrá verdad pero será una verdad que alberga en su seno la posibilidad del error.

La verdad que no albergue al error es por tanto una verdad incompleta, y no reconocer esto, es caer en la ilusión de una intelección computante capaz de abarcarlo todo; una omnisciencia específica para un objeto concreto. Desligar al sujeto y a sus 
operaciones del gran teorema del conocimiento es desconocer lo que de falible éste tiene y lo que de su falibilidad puede aportar a su abordaje al objeto para computarlo.

Así como orden y desorden no se pueden aislar en unidades separadas e independeintes; así mismo, el concepto de verdad y error tampoco, ya que son equivalentes de estos y comportan relaciones complejas: complementarias y antagónicas al mismo tiempo. Recurrir a uno sin posibilitar la entrada del otro a la ecuación paradigmática del conocimiento, es dejar de lado las facetas de aleatoriedad, de azar, que son cotidianos a los cuales el hombre se enfrenta. Verdad-error como función resultante del cómputo vivo de la información, no es tampoco la fórmula mágica del universo, simplemente resulta de la interacción funcional y constitutiva de la physis que se pone en relación estrecha con las dimensiones abstractas del pensamiento dentro del campo biológico.

Separar lo físico de lo biológico y de lo antroposocial, es caer en una reducción peor o igual a aislar la verdad del error, ya que las relaciones de estas tres, van a determinar el punto de partida sin el cual ningún conocimiento sería posible. Ruidos, azares, aleas, indeterminaciones; son los caminos por donde el hombre avanza. Sin embargo, no se expresa que se trate de un panegírico del desorden dándole primacía al mismo sobre cualquier otra realidad; lo que se evidencia es la necesidad de reconocer lo que de enigmático y confuso hay en el conocimiento para no asumir falsas presunciones de infalibilidad. El fenómeno del conocimiento se camufla detrás de bambalinas, detrás de sombras de confusión, azar e indeterminación, o sea, detrás de las limitaciones constitutivas de la biología humana.
Se tendrían que enseñar principios de estrategia que permitan afrontar los riesgos, lo inesperado, lo incierto, $\mathrm{y}$ modificar su desarrollo en virtud de las informaciones adquiridas en el camino. Es necesario aprender a navegar en un océano de incertidumbres a través de archipiélagos de certeza (Morin, 1999, p. 3).

\section{CONCLUSIONES}

La complejidad como una emergencia subordinada de las dinámicas ordenadoras de los sistemas vivos, va más allá de la complicación ya que terminológicamente se diferencian en el grado de sentido, pero se compromenten en el grado de recursión. La complejidad como método, es decir, como camino experiencial hacia la comprensión del comportamiento de la diversidad que interactúa, puede pensarse no como un camino absolutamente necesario hacia la verdad, sino meramente como una herramienta que vale la pena analizar en cuanto a la potencialidad que entraña para llegar a adquirir conciencia de conocimiento, a adquirir conciencia del ocultamiento de los fenómenos bajo la especie de ruidos informacionales que impiden conocer a los objetos de estudio de manera plena.

Consistente en el hecho de la imposibilidad de existencia de orden separado del dato de desorden, hay también una imposibilidad de existencia en el plano del conocimiento de verdades que no estén relacionadas con el error, en una u otra forma; apoyado esto en el hecho del tratamiento informacional que hace el cerebro de los datos para constituir la computación y por ésta la cogitación. 
Ello refleja la urgencia por cambiar los conceptos epistemológicos fundados en el orden de las verdades absolutas que son captadas por el intelecto humano. El objeto que sustenta estas verdades como tal existe, pero en cuanto se hace referencia a una verdad extraída de los mismos, debido a los mecanismos falibles de extracción de la información, hacen difícil la datación de las mismas. Es necesario por tanto repensar la ciencia. Ésta debe ser en primera medida una ciencia para el hombre. Todo esfuerzo por apropiarse del conocimiento del mundo, se convierte en un imperativo junto a la necesidad transdiciplinaria de pensar al hombre para llegar a un sano concepto de desarrollo fundado más allá de lo económico, científico, técnico y tecnológico (impulsores estos de la sociedad moderna)

\section{BIBLIOGRAFÍA}

Barandiaran, X. (S.A.). Resumen y comentarios al "Árbol del conocimiento". Recuperado el 05 de 12 de 2006, de $\mathrm{http} / / /$ sindominio.net/autonomiasituada/t extos/matvar/matvar.pdf

Bertalanfy, L. (1986). Teoría general de los sistemas. México D.F.: Fondo de Cultura Económica.

Bloom, W., \& Fawcett, W. (1973). Tratado de histología. Buenos Aires: Labor.

Ciurana, E. (S.F.). Complejidad: elementos para una definición. Recuperado el 2007, de http://www.pensamientocomplejo.com.a r

Córdoba García, F. (2005). Fundamentos biológicos del aprendizaje y la memoria. Recuperado el 2016, de http://www.uhu.es/francisco.cordoba/asi gnaturas/FBAM/TEMAS\%20PDF/3LA\%20NEURONA.pdf
Darwin, C. (1899). Viaje de un naturalista al rededor del mundo. Madrid: Madrid: La España Moderna.

Darwin, C. (2005). El origen del hombre. México D.F. : Época.

Descartes, R. (1986). Discurso del método. Bogotá: Panamericana.

Dostoievski, F. M. (1945). Crimen y castigo. Buenos Aires: W. M. Jackson.

Dúc Tháo, T. (1959). Fenomenología y materialismo dialéctico. Buenos Aires: Lautaro.

García, B. E. (S.F.). La teoría de la educación en Niklas Luhmann. Recuperado el 2006, de www.oei.es/oeivirt/salacredi/bEATRIZ. pdf

Gödel, K. (2006). Sobre proposiciones formalmente indecidibles de los principia mathematica y sistemas afines I. Brünn: KRK Ediciones.

Gómez García, P. (2003). La antropología compleja de Edgar Morin. Granada: Imprenta Santa Rita.

Kafka, F. (1969). La metamorfósis; Un artista del hambre; Un artista del trapecio. Madrid: Alianza.

Kafka, F. (1986). La metamorfósis. Madrid: Alba.

López Ramírez, O. (1998). El paradigma de la complejidad en Edgar Morin. Revista del Departamento de Ciencias Universidad Nacional sede Manizales, 98-114.

Martínez Miguélez, M. (2007). Conceptualización de la transdisciplinariedad. POLIS, Revista Latinoamericana, 1-17.

Martínez Miguélez, M. (S.F.). Transdisciplinariedad y lógica 
dialéctica. Conciencia Activa 1, 107146.

Moray, N. (1967). Cibernética. Barcelona: Editorial Herder.

Morin, E. (1984). Ciencia con conciencia. Barcelona: Gerder.

Morin, E. (1992). El método IV: las ideas. Madrid: Cátedra.

Morin, E. (1993 y 1995). Civilizar: La nueva conciencia planetaria. En E. Morin, Tierra Patria y Mis demonios.

Morin, E. (1999). El método III: el conocimiento del conocimiento. Madrid: Cátedra.

Morin, E. (1999). Los siete saberes necesarios para la educación del futuro. París: UNESCO.

Morin, E. (2001). El método: La naturaleza de la naturaleza. Madrid: Cátedra.

Morin, E. (2001). Introducción al pensamiento complejo. Barcelona: Gerder.

Morin, E. (2002). El método II: la vida de la vida. Madrid: Cátedra.

Morin, E. (2003). El método V: la humanidad de la humanidad. Madrid: Cátedra.

Morin, E. (2007). Edgar Morin: El pensador planetario de las luciérnagas más luminosas. Recuperado el 2007, de http://www.edgarmorin.org/LinkClick.a spx $?$ link $=57 \&$ mid $=435$

Morin, E. (S.F.). Sobre la interdisciplinariedad. Recuperado el 2005, de http://www.carlosberzunza.org/images/ morin_sobre_la_interdisciplinaridad.pdf

Morin, E., \& Le Moigne, J. L. (2006). Inteligencia de la complejidad: Epistemología y pragmática. S.C.: l'aube.

Morin, E., Ciurana, E. R., \& Motta, R. D. (2002). Educar en la era planetaria: El pensamiento complejo como método de aprendizaje en el error y la incertidumbre humana. Salamanca: UNESCO; Universidad de Valladolid; Instituto Internacional para el Pesnamiento Complejo (IIPC); Universidad del Salvador (USAL) .

Murray, R., Bender, D., Botham, K., Kennelly, P., Rodwell, V., \& Weil, A. (2000). Harper Bioquímica. México D.F.: Mc Graw Hill.

Najmanovich, D. (2000). Desamurallar la educación: hacia nuevos paisajes educativos. Recuperado el 2006, de http://aprendeenlinea.udea.edu.co/lms/m oodle/file.php/33/Denise_Najmanovich_ -_Desamurallar_la_Educacion.pdf

Najmanovich, D. (2001). Del cuerpo máquina al cuerpo entramado. Campo grupal, 2935.

Najmanovich, D. (2005). El juego de los vínculos: subjetividad y redes: figuras en mutación. Buenos Aires: Biblos.

Oparin, A. (1997). El origen de la vida. Santa Fé de Bogotá: Ediciones Emfasar.

Schurzbok, E. (S.A.). Modelo universal de la mente. Recuperado el 2004, de http://axxon.com.ar/zap/czapping0142.htm

Serrano, J. (1986). La reducción en las ciencias. Estudios de Filosofía, Arte, Letras.

Tarski, A. (S.A.). La concepción semántica de la verdad y los fundamentos de la semántica. A parte Rei. Revista de filosofía, 1-30.

Wheatley, M., \& Kellner Rogers, M. (1998). A simpler way. Berkeley: Publishers Group West. 